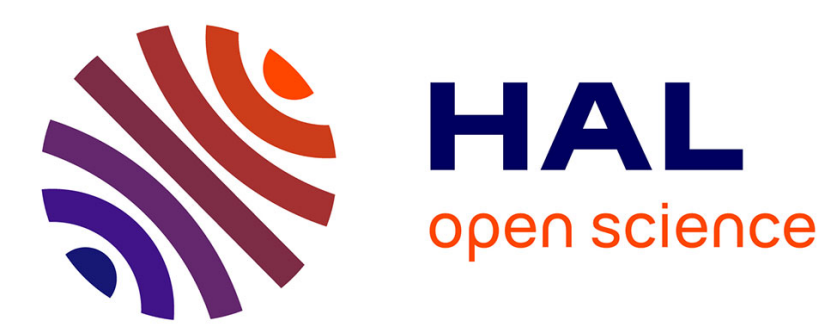

\title{
Waveform transition in the transient focusing field
}

\author{
T. Otani, H. Tominaga
}

\section{To cite this version:}

T. Otani, H. Tominaga. Waveform transition in the transient focusing field. Journal de Physique IV Proceedings, 1994, 04 (C5), pp.C5-733-C5-736. 10.1051/jp4:19945158 . jpa-00252837

\section{HAL Id: jpa-00252837 https://hal.science/jpa-00252837}

Submitted on 1 Jan 1994

HAL is a multi-disciplinary open access archive for the deposit and dissemination of scientific research documents, whether they are published or not. The documents may come from teaching and research institutions in France or abroad, or from public or private research centers.
L'archive ouverte pluridisciplinaire HAL, est destinée au dépôt et à la diffusion de documents scientifiques de niveau recherche, publiés ou non, émanant des établissements d'enseignement et de recherche français ou étrangers, des laboratoires publics ou privés. 


\title{
Waveform transition in the transient focusing field
}

\author{
T. OTANI and H. TOMINAGA
}

Department of Electrical Engineering, Faculty of Engineering, Doshisha University, Kamigyo-ku, 602 Kyoto, Japan

\begin{abstract}
In classical optics and acoustics, the focusing field for harmonic or monochromatic waves is characterized by a phenomenon of wave concentration and phase anomaly. For the transient focusing field, significant waveform deformation or waveform transition takes place in the vicinity of the focus. It is shown here theoretically and experimentally that the focusing of the transient wave is characterized by time reversal and polarity inversion effects.
\end{abstract}

\section{INTRODUCTION}

The focusing of acoustic waves have long been a classical topic of theoretical and experimental acoustics. Focused ultrasonic fields are nowadays commonly used in acoustical imaging applications to improve the lateral resolution in non-destructive testing, medical diagnosis as well as in acoustic microscopy. Highly focused fields have also raised interest in the acoustical evaluation of the micro structure of solid materials. In this method, the materials to be evaluated are characterized by the acoustical boundary response to the incident wave after scattering, reflection and transmission. It is thus indispensable to understand the exact nature of the incident wave or the focusing field for proper characterization to be possible.

Focusing is generally understood by the phenomenon of wave concentration and phase shifting by $-\pi$ radians at the focal region. This is known as the phase anomaly and is explained by the effect of wave diffraction in monochromatic wave fields. In transient wave fields, the singularity of focusing is observed as a waveform transition along the focusing axis and near the focal point. The theoretical impulse response of the focusing pressure field was introduced with some approximations by Penttinen and Luukkala. ${ }^{1)}$ Djelouah, Baboux and Pedrix studied theoretically and experimentally the transient focusing field under different types of source excitation and pointed out a polarity inversion between the geometrical wave and the diffracted wave across the focus. ${ }^{2}{ }^{2}$ This remark is easily recognized by off-axis pressure waveforms but not easily recognized by on-axis waveforms. We clarify here the physics of focusing by the use of a focusing source with a weighted velocity distribution.

\section{THEORETICAL EXPRESSION OF FOCUSING ACOUSTIC FIELD ${ }^{1-4)}$}

For a planar source $S$ mounted in an infinite rigid baffle, the velocity potential can be derived from Green's theorem and expressed by the Rayleigh integral:

$$
\phi=\frac{1}{2 \pi} \iint_{s} \frac{u(t-R / c)}{R} d S
$$

where $u(t)$ is velocity normal to source surface.

For non-planar sources, this expression is not exact because secondary diffraction is not taken into account. As many authors pointed out, the Rayleigh integral gives a good approximate evaluation of the radiated field on the condition that secondary diffraction can be neglected. Figure 1 shows the geometry of a spherically concave source defined by radius $a_{0}$, curvature $R_{0}$ and half aperture angle $\alpha$ Supposing the source surface $S$ with a uniform distribution of velocity $u(t)$ is only slightly curved and the secondary diffraction can be ignored, the acoustic pressure on the symmetrical axis is expressed by

$$
p=\rho \frac{\partial \phi}{\partial t}=\rho c\left(\frac{R_{0}}{R_{0}-R_{1}}\right) \int_{\tau_{1}}^{\tau_{2}} \frac{\partial}{\partial \tau} u(\tau) d \tau=\rho c\left(\frac{R_{0}}{R_{0}-R_{1}}\right)\left[u\left(\tau_{1}\right)-u\left(\tau_{2}\right)\right]
$$


where $\tau=t-R / c, \tau_{1}=t-R_{1} / c$ and $\tau_{2}=t-R_{2} / c$.

By using $\Delta \tau \equiv\left|\tau_{2}-\tau_{1}\right|=\left|R_{2} / c-R_{1} / c\right|$, the acoustic pressure on the axis is defined by, in region $\mathrm{I}\left(R_{1}<R_{0}\right)$

$$
p=\rho c \frac{R_{0}}{\left|R_{0}-R_{1}\right|}\left[u\left(\tau_{1}\right)-u\left(\tau_{1}-\Delta \tau\right)\right]
$$

in region II $\left(R_{1}>R_{0}\right)$,

$$
p=-\rho c \frac{R_{0}}{\left|R_{0}-R_{1}\right|}\left[u\left(\tau_{1}\right)-u\left(\tau_{1}+\Delta \tau\right)\right]
$$

and at the focal point $\left(R_{1}=R_{0}\right)$,

$$
p_{0}=\left.p\right|_{R=R_{0}}=\lim _{R_{1} \rightarrow R_{0}} p=\rho h \frac{\partial}{\partial \tau_{0}} u\left(\tau_{0}\right)=\rho h a\left(\tau_{0}\right)
$$

where $h$ is the depth of the concave surface $a(t)=\partial u(t) / \partial t$ (source acceleration) and $\tau_{0}=t-R_{0} / c$. Therefore, from eq. (3) and (4), one may reduce that the focusing process across the focus is a combination of both time reversal and polarity inversion effects. For a focusing source having an axisymmetric surface velocity distribution, the acoustic pressure is also expressed as

$$
p= \begin{cases}\rho c \frac{R_{0}}{\left|R_{0}-R_{1}\right|}\left[u_{g}\left(\tau_{1}\right)-u_{d}\left(\tau_{1}-\Delta \tau\right)\right] & \text { for } R_{1}<R_{0} \\ -\rho c \frac{R_{0}}{\left|R_{0}-R_{1}\right|}\left[u_{g}\left(\tau_{1}\right)-u_{d}\left(\tau_{1}+\Delta \tau\right)\right] & \text { for } R_{1}>R_{0}\end{cases}
$$

where $u_{g}$ and $u_{d}$ are the geometrical and the diffracted waves respectively and can be specified according to the velocity distribution on the surface and its time dependent function.

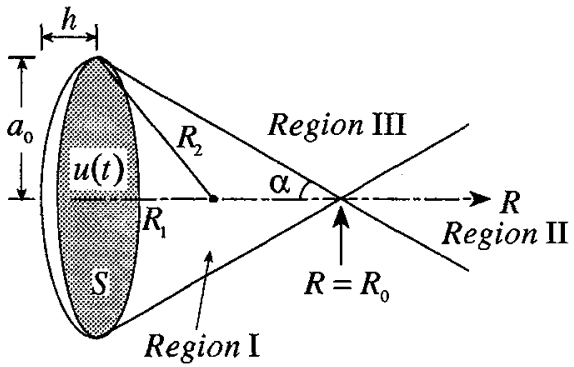

Fig. 1 Geometry and notation of a concave focusing source.

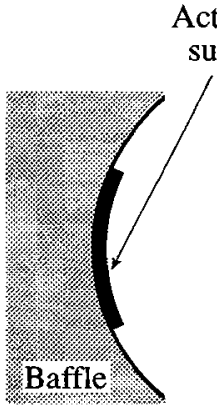

(a)
Active surface

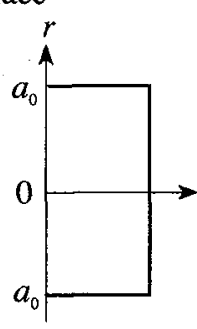

(b)

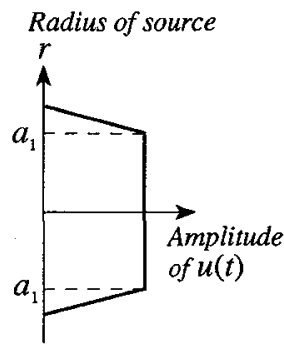

(c)
Fig. 2 Velocity distribution of source surface; (a) a concave focusing surface, (b) uniform velocity distribution, (c) weighted velocity distribution.

\section{NUMERICAL SIMULATION AND EXPERIMENTAL RESULTS}

\subsection{Numerical simulation}

For numerical simulation and experimental verification focusing concave and circular transducers with an active radius $a_{0}=10 \mathrm{~mm}$ and curvature radius $R_{0}=100 \mathrm{~mm}$ were used. For numerical simulation we applied, as the source acceleration, the Dirac delta function and the sinusoidal impulse (one period of sinusoidal wave). Medium constant were $\rho=1 \mathrm{~kg} / \mathrm{m}^{3}$ and $c=1495 \mathrm{~m} / \mathrm{s}$. Figure 3 (a) and (b) show calculated pressure waveforms on the axis at $R_{1}=50 \mathrm{~mm}$ in region $\mathrm{I}, R_{1}=50 \mathrm{~mm}$ at the focal point and $R_{1}=150 \mathrm{~mm}$ in region II. These simulated waveforms include the focusing effects of the time reversal and 
the polarity inversion. However, it is not easy to recognize the effects in Fig. 3. To clarify the time reversal and polarity inversion effects of the focusing, one may use a velocity distribution on the source surface to reduce the diffraction from the circumference edge of the surface. We assumed for numerical simulation a ramped velocity distribution of the source as shown in Fig. 2, a uniform velocity inside the radius $a_{1}$ and linearly decreasing velocity from $a_{1}$ to the outer edge $a_{0}\left(a_{1}=7 \mathrm{~mm}\right.$ and $\left.a_{0}=10 \mathrm{~mm}\right)$. Figure 4 shows the calculated pressure waveforms on the axis radiated from the ramped velocity distribution source. The waveforms in Fig. 4 show clearly the time reversal effect between region I and region II (in front of the focus and behind the focus). In the case where the acceleration has a waveform defined by the Dirac delta function, the polarity inversion effect is not easily recognized (Fig. 3 (a)). In this case, the pressure waveforms in region I (in front of the focus) can be interpreted by using eq. (5) as the sum of the geometrical wave in the positive step function and the diffracted wave in the negative ramped step function as shown in Fig. 5. Then, in region II (behind the focus), the pressure waveforms can be explained by an exchange of the arrival time between the two waves (the time reversal effect) and by an inversion of polarity. Thus it is found that the effects of time reversal and polarity inversion are valid in the case of the delta function. In the case of the sinusoidal impulse acceleration, the time reversal and the polarity inversion effects are clearly shown in Fig. 4 (b).
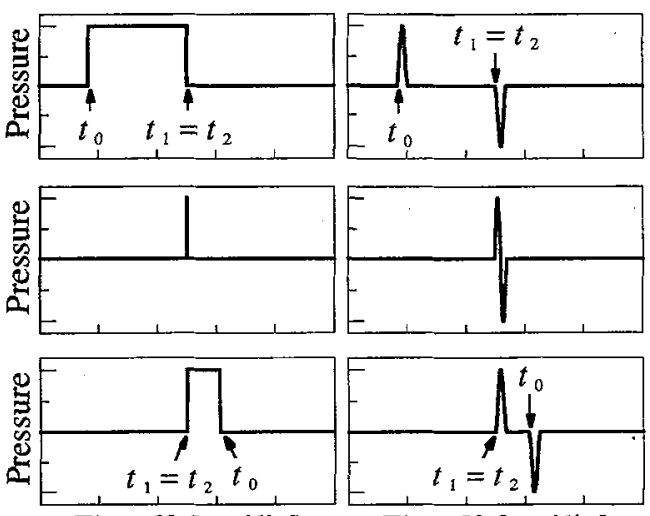

Time $[0.2 \mu \mathrm{s} / \mathrm{div}]$

(a)

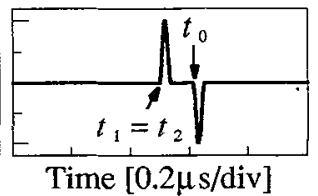

(b)

Fig. 3 Pressure waveforms on the axis with uniform velocity distribution; (a) delta function acceleration, (b) sinusoidal impulse acceleration.
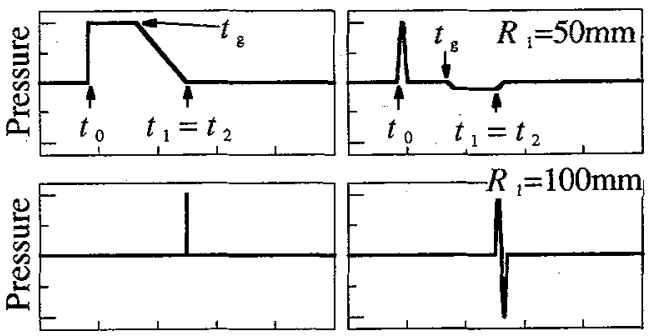

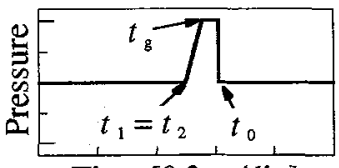

Time $[0.2 \mu \mathrm{s} / \mathrm{div}]$

(a)

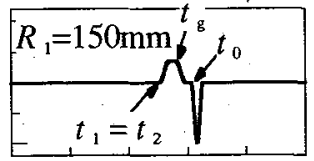

Time $[0.2 \mu \mathrm{s} / \mathrm{div}]$

(b)
Fig. 4 Pressure waveforms on the axis with weighted velocity distribution; (a) delta function acceleration, (b) sinusoidal impulse acceleration.

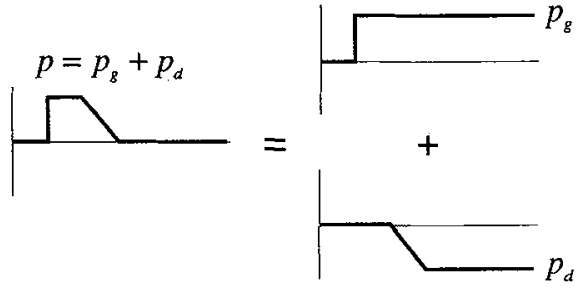

(a) Region I $\left(R_{1}<R_{0}\right)$

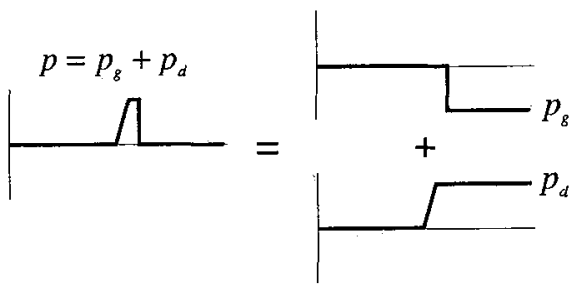

(b) Region $\mathrm{II}\left(R_{1}>R_{0}\right)$

Fig. 5 Formation of pressure waveforms on the axis in focusing field. 


\subsection{Experimental results}

Measurements of focusing fields were carried out to verify the above observation on the focusing. Concave focusing sources were made by piezoelectric polymer films (P(VDF-TrFE)) of diameter 10mm and thickness $35 \mu \mathrm{m}$, placed on the center of a concave baffle by adhesive. To realize the velocity distribution on the source surface, the active surface (piezoelectric film) is trimmed in the shape of a star. The acoustic pressure was measured by using a miniature PVDF ultrasonic hydrophone probe $(0.6 \mathrm{~mm}$ diameter) supplied by MEDICO TEKNISK INSTITUT. Pressure waveforms were again observed at $R_{1}=50 \mathrm{~mm}$ in region I, at $R_{1}=100 \mathrm{~mm}$ at the focus and $R_{1}=150 \mathrm{~mm}$ in region II. Fig. 6 (a) and Fig. 7 (a) show the results when a unit step voltage was applied to the source. At the focus, the pressure waveforms are approximately the sinusoidal impulse, which is regarded as the source acceleration waveform. Using these results, we estimated the impulse response of this focusing filed on the axis by a deconvolution method (Fig. 6 (b) and Fig. 7 (b)) which corresponds to the calculated impulse response of Fig. 3 (a) and Fig. 4 (a). The frequency band is limited at $8 \mathrm{MHz}$ in the inverse calculation. It seems that the observed waveforms in region I and in region II are acceptable by comparison to the calculated waveforms of Fig. 3 and Fig. 4.
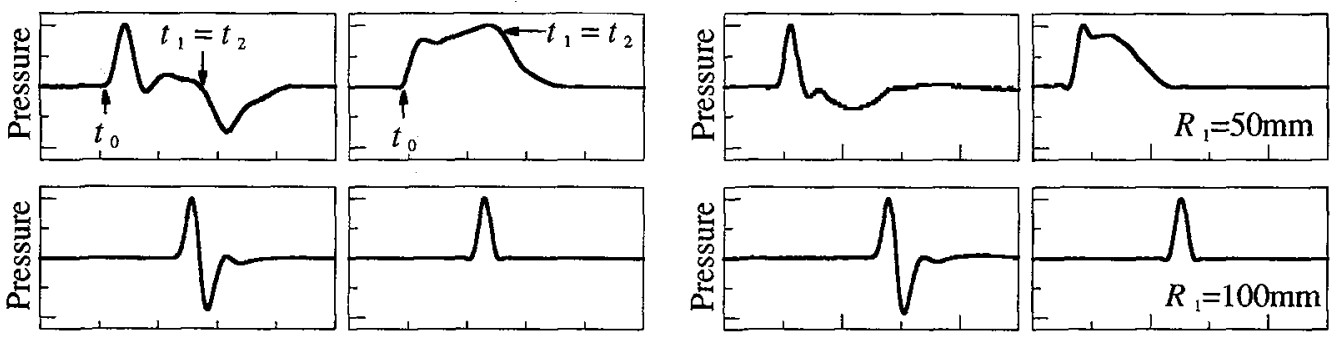

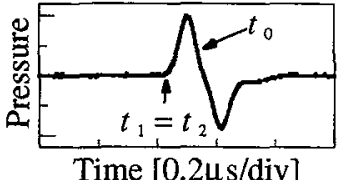

(a)

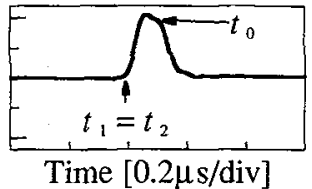

(b)

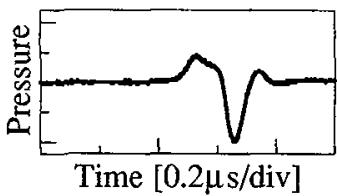

(a)

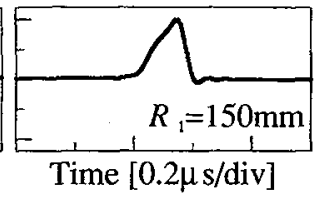

(b)

Fig. 6 Experimental results with uniform velocity distribution; (a) observed pressure waveforms on the axis, (b) estimated impulse responses on the axis.

Fig. 7 Experimental results with weighted velocity distribution; (a) observed pressure waveforms on the axis, (b) estimated impulse responses on the axis.

\section{CONCLUSIONS}

The time reversal and the polarity inversion effects of the focusing field of the axis is explained by both numerical simulation and experimental observations. This remark is also valid for the off-axis in region I and region II. As described in classical optics and acoustics, the focusing field is characterized by a wave concentration and a face jump $\pi$ of the incident wave as it passes through the focus. This expression is appropriate for a harmonic or monochromatic wave field. For the transient focusing field in time domain form, the phenomenon of focusing may be described as follows: "In the geometrical wave zone in region I and in region II, the focusing is characterized by the time reversal (the time reversal of the geometrical wave and the diffracted wave) and by the polarity inversion effect between the two waves." In the shadow zone, in region III, no reversal and inversion effects exist.

\section{REFERENCES}

[1] Penttinen, A. and Luukkala, M., J. Phys. D Appl. Phys. 9 (1976) 1547-1557.

[2] Djelouah, H., Baboux, J. C. and Perdrix, M., Ultrosonics 29 (1991) 188-200.

[3] Hamilton, M. F., J. Acoust. Soc. Am., 92 (1992) 527-532.

[4] Fro $\phi y s a$, K. -E., Naze Tjфtta, J. and Tjфtta, S., J. Acoust. Soc. Am. 93 (1993) 80-91. 